Orthopäde 2011 · 40:971-977

DOI 10.1007/s00132-011-1825-3

Online publiziert: 20. Oktober 2011

(c) Springer-Verlag 2011
V. Valderrabano · A. Frigg · A. Leumann · M. Horisberger

Abteilung Orthopädie, Universitätsklinikum Basel

\title{
Sprunggelenkprothese bei Valgusarthrose
}

Das obere Sprunggelenk (OSG) und der Rückfuß sind ein biomechanisch hochkomplexes Konstrukt, dem mit einem prothetischen Ersatz zu genügen schon bei physiologisch korrektem Alignement des Rückfußes außerordentlich schwierig ist. Noch schwieriger wird dies, wenn die Begleitfaktoren bei einer Prothesenversorgung nicht dem biomechanischen Normalfall bzgl. Achsen und Stabilität entsprechen. Die Implantation einer Sprunggelenkprothese ist bei vorbestehender Arthrosefehlstellung technisch anspruchsvoll. Wird die Fehlstellung bei der prothetischen Versorgung nicht oder ungenügend korrigiert, muss mit einem Versagen der Prothese gerechnet werden.

Die vorliegende Übersicht soll eine kurze Zusammenfassung liefern, wie die Valgusfehlstellung entsteht, wie trotzdem meistens erfolgreich eine Sprunggelenkprothese implantiert werden kann und was die möglichen Fallstricke sein können.

\section{Ursachen der \\ Valgus-OSG-Arthrose}

Das Alignement des OSG wird analog zum Knie in ein supramalleoläres („Femur“), tibiotalares („Kniegelenkspalt“) und inframalleoläres („Tibia“) Alignement unterteilt $[12,13]$. Das supramalleoläre und tibiotalare Alignement können gut und sicher mit a.-p.-Röntgenbildern und bei Bedarf Orthoradiogrammen erfasst werden. Schwieriger ist es, das inframalleoläre Alignement zu bestimmen:

- die klinische Messung von Auge und mit Goniometer ist ungenau $[1,4,13$, 27];

- bei einer konventionellen a.-p.-Röntgenaufnahme des OSG überlagert der Mittelfuß die Ferse und durch die $20^{\circ}$-Innenrotation wird die Ferse nach lateral gedreht und täuscht einen Valgus vor.

Deshalb ist eine spezielle Rückfußaufnahme wie z. B. nach Saltzman erforderlich: dies erlaubt, das Verhältnis der mechanischen Achse des Rückfußes unter Belastung zur Tibia darzustellen [12, 13, 20]. Die wichtigsten Kriterien für die Einstellung einer korrekten OSG-Biomechanik in der Frontalebene sind

- ein tibiotalarer Winkel von ca. 90 ${ }^{\circ}$ [19] und

- eine neutrale Rückfußstellung [13, 20].

Es ist zu beachten, dass in der SaltzmanAufnahme der gesunde Rückfuß in neutraler bis minimaler Varusstellung von $1-2^{\circ}$ steht und nicht wie allgemein angenommen in einem Valgus von $5-7^{\circ}[3,8$, $12,13,17,18,20]$.

Werden diese physiologischen Werte mit vermehrtem Valgus überschritten, führt dies zu einer Druckzunahme
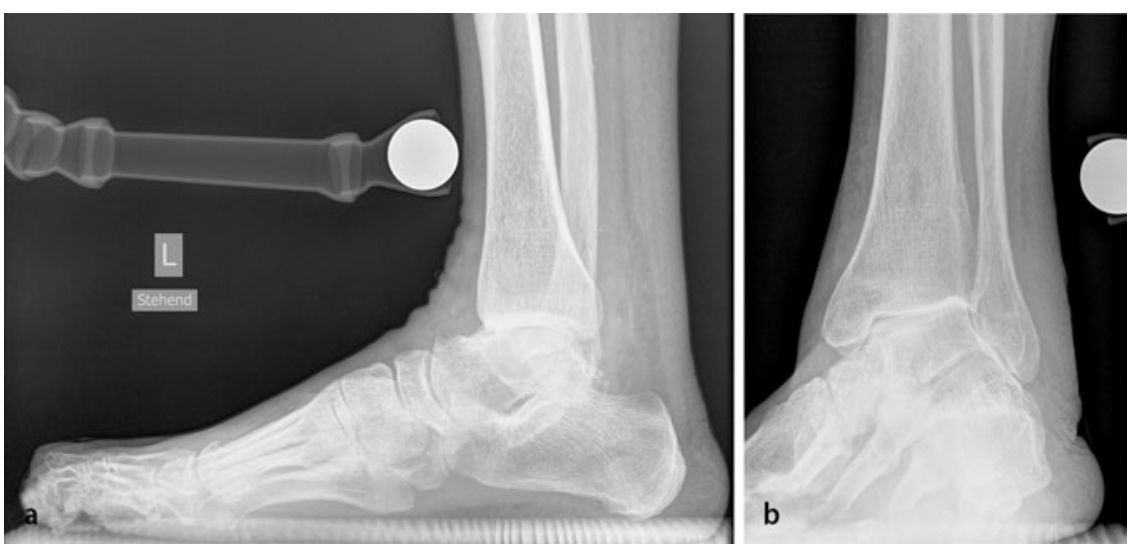

Abb. 1 A Ligamentär bedingte OSG-Valgusarthrose. Der 83-jährige Patient hatte aufgrund einer medialen OSG-Instabilität eine Valgusarthrose entwickelt. Die Bilder zeigen die Situation vor der Prothesenimplantation. a Seitliche, b a.-p.-Projektion im Stehen. OSG oberes Sprunggelenk im lateralen OSG. Der lateralisierte Zug des Triceps surae unterstützt die schrittweise Destabilisierung, den asymmetrischen Knorpelverschleiß im lateralen OSG und tibiofibulären Gelenk sowie die Stressbelastung der Syndesmose. Es entwickelt sich schließlich eine generalisierte Valgusarthrose des OSG. In einer großen Untersuchung von OSG-ArthrosePatienten wiesen präoperativ $8 \%$ aller $\mathrm{Pa}$ tienten einen Valgus von $>10^{\circ}$ auf [24]. Über die genaue Rolle der reinen Valgusfehlstellung bei der Entstehung einer OSG-Arthrose gibt es unseres Wissens keine Literatur. Wohl ist aber belegt, dass eine mediale OSG-Bandinstabilität, die in einer Untersuchung von ligamentär bedingter OSG-Arthrose in sämtlichen Fällen mit einer Valgusfehlstellung vergesellschaftet war, zu einer OSG-Arthrose führen kann [23]. Clarke et al. [5] zeigten in einer In-vitro-Studie, dass die Durchtrennung des medialen Bandapparats die Gelenkkontaktfläche um 15-20\% verringerte. Dies stimmt mit der Beobachtung anderer Autoren überein, dass die chronische 

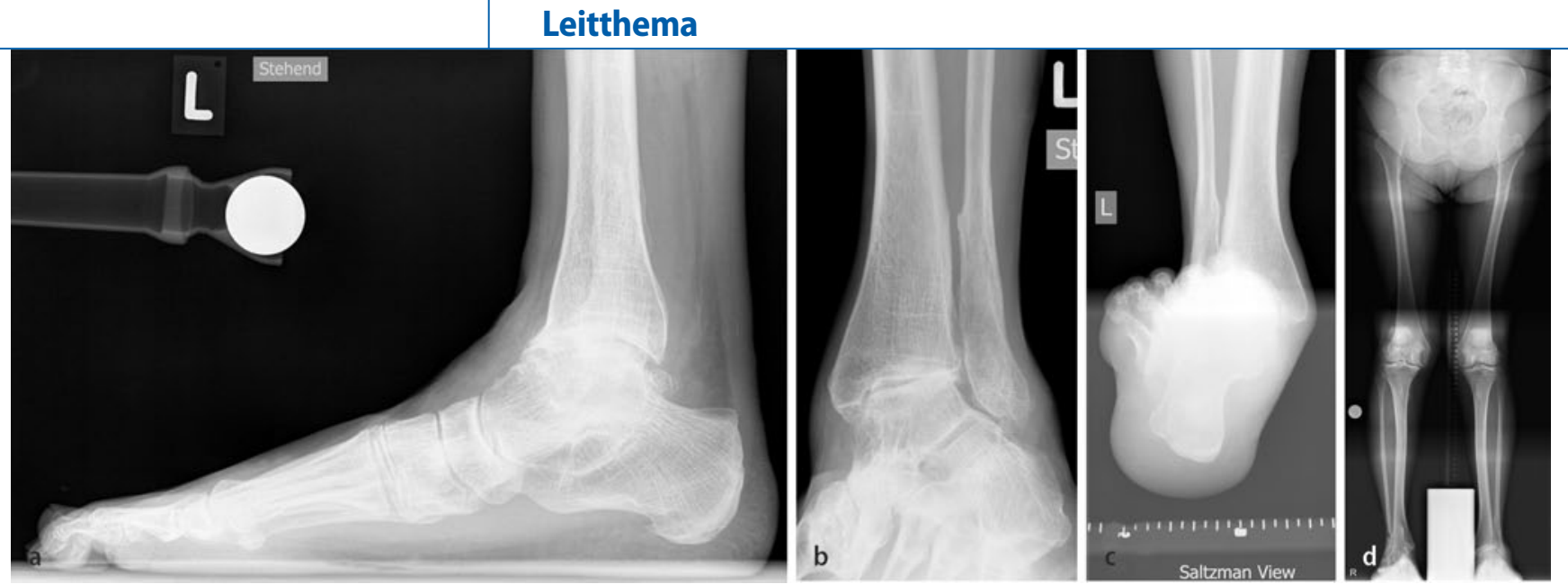

Abb. $2 \Delta$ Posttraumatische Valgus-OSG-Arthrose. Die 69-jährige Patientin zog sich einige Jahre zuvor eine in Fehlstellung verheilte Malleolarfraktur Typ Weber B zu. In der Folge kam es zu einem Valguskollaps des Rückfußes mit OSG-Degeneration und Entwicklung eines sekundären Pes planovalgus. Zudem fand sich ein Genu valgum auf der betroffenen Seite. $\mathbf{a}$ Seitliche, $\mathbf{b}$ a.p.-Projektion im Stehen, c Saltzman Aufnahme zur Beurteilung des Rückfußalignements, d Orthoradiogramm. OSG oberes Sprunggelenk
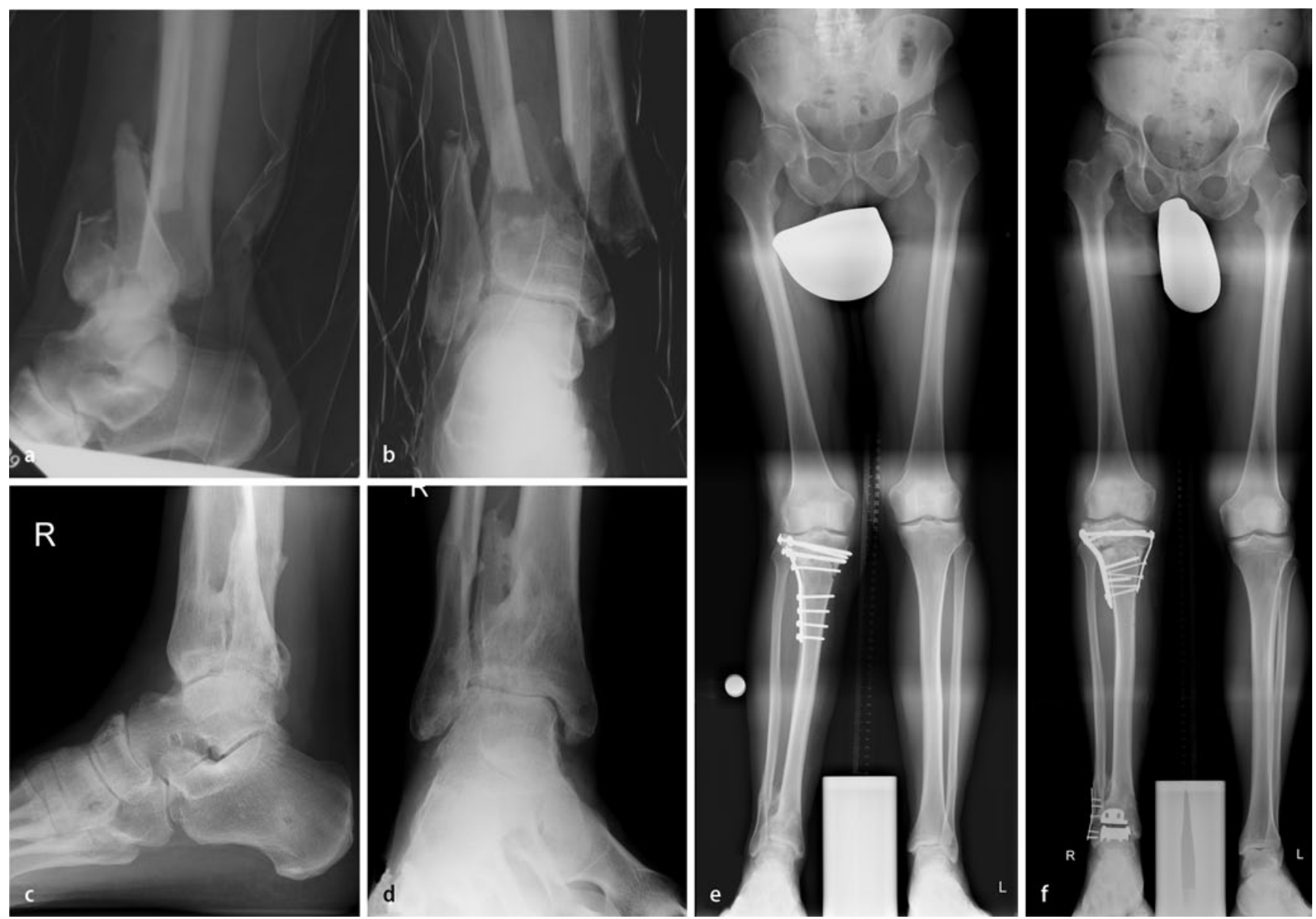

Abb. $3 \Delta$ Valgus-OSG-Arthrose. OSG-Prothese und Beinachsenkorrektur. Der 45-jährige Patient erlitt 3 Jahre zuvor eine offene distale Unterschenkelfraktur und vor 2 Jahren eine Tibiaplateaufraktur auf der gleichen Seite. Beides resultierte in einer Valgusdeviation. Um ein gutes Ergebnis der prothetischen Versorgung am OSG zu erreichen, müssen beide Lokalisationen der Deformität in einem Schritt angegangen werden: OSG-Prothese und proximale Tibiaosteotomie. a, b Offene distale Unterschenkelfraktur; c, d Entwicklung einer endgradigen, lateral betonten OSG-Arthrose innerhalb kurzer Zeit; d, e Orthoradiogramm vor und nach Korrektur der Fehlstellung. OSG oberes Sprunggelenk 
mediale Instabilität mit Valgusfehlstellung zur frühzeitigen Arthrosebildung prädisponiert [9] und diese einen raschen Verlauf nimmt (• Abb. 1; [23]).

( ) Eine mit einer Valgusfehlstellung vergesellschaftete mediale OSG-Bandinstabilität kann zu einer OSG-Arthrose führen

$\mathrm{Zu}$ diesem Formenkreis zählt auch der schwere Pes planovalgus et abductus auf dem Boden einer Tibialis-posterior-Sehneninsuffizienz. Ihr Beitrag zur Entstehung einer OSG-Arthrose ist noch weitgehend unklar. Es ist jedoch davon auszugehen, dass eine schwere Plattfussdeformität (Stadium IV) über analoge Mechanismen zu einer OSG-Arthrose führt [2].

Eine andere Ursache der Valgusfehlstellung sind ossäre Deformitäten. Diese treten z. B. nach in Verkürzung ausheilenden Fibulafrakturen (z. B. nach Weber-Boder-C-Frakturen) oder Stauchung des lateralen Anteils der Tibiaplateaus (z. B. bei einer Pilon-Fraktur) mit folgender Osteonekrose und Valgusfehlstellung auf [16]. Neuere biomechanische Untersuchungen zeigten, dass posttraumatische Achsabweichungen des distalen Unterschenkels zu signifikanten Änderungen der Kontaktfläche im Sprunggelenk mit zunehmender Achsabeichung und Nähe zum Gelenk führen [21, 22].

\section{OSG-Prothesen bei gleichzeitiger Korrektur der Valgusfehlstellung}

Nach Ausschöpfung gelenkerhaltender Therapieoptionen, die beispielsweise eine Umstellungsosteotomie der distalen Tibia oder des Rückfußes sowie Bandplastiken beinhalten [19], bleiben als Optionen lediglich die Implantation einer OSG-Prothese oder eine OSG-Arthrodese. Hierbei ist zu berücksichtigen, dass eine OSGProthese nur dann implantiert werden sollte, wenn eine korrekte und vollständige Normalisierung der Biomechanik bzgl. Gelenkachse und Stabilität mit zusätzlichen operativen Maßnahmen erreicht werden kann. Haskell u. Mann [14] haben nachgewiesen, dass bei einer präoperativen Fehlstellung in der Frontalebenen von $<10^{\circ}$ ein gutes Ergebnis erwartet

Orthopäde 2011 · 40:971-977 DOI 10.1007/s00132-011-1825-3

(C) Springer-Verlag 2011

V. Valderrabano · A. Frigg · A. Leumann · M. Horisberger Sprunggelenkprothese bei Valgusarthrose

Zusammenfassung

Die Arthrose des oberen Sprunggelenks (OSG) ist häufig mit einer Fehlstellung verbunden, dabei ist die Valgusarthrose seltener als die Varusarthrose. Die Ursachen für eine Valgusarthrose sind eine mediale Bandinstabilität, ein Pes planovalgus und posttraumatische Fehlstellungen z. B. nach Fibulafraktur oder Stauchung des lateralen Tibiaplateaus. Damit eine OSG-Prothese kurz- wie auch langfristig korrekt und schmerzfrei funktionieren kann, muss die Biomechanik entsprechend den Prinzipien der mechanischen Achse, deren Wichtigkeit in der Orthopädie allgemein akzeptiert ist, wiederhergestellt werden. Richtlinien sind dabei (1) ein anteriorer tibiotalarer Winkel von etwa $90^{\circ}$ und (2) eine neutrale Rückfußstellung. Diese wird vor- zugsweise mit der Rückfußaufnahme nach Saltzman gemessen. Dabei ist zu beachten, dass der normale Rückfuß in einer Neutralstellung bis $1-2^{\circ}$ Varusposition und nicht wie bisher angenommen in einer Valgusstellung ist. Je nach Ausmaß und Lokalisation der Valgusdeformität werden in unterschiedlicher Reihenfolge (1) die OSG-Prothese implantiert, (2) supra- und (3) inframalleoläre Korrekturosteotomien/-Arthrodesen, (4) eine mediale Bandplastik, (5) eine Fibulaosteotomie (6) mit eventueller Rekonstruktion der Syndesmose durchgeführt.

\section{Schlüsselwörter} Sprunggelenkprothese - Valgusarthrose . Osteotomie $\cdot$ Bandplastik $\cdot$ Arthrodese

\section{Total ankle arthroplasty in valgus ankle osteoarthritis}

\section{Abstract}

Ankle osteoarthritis (OA) is often associated with deformities. Valgus $O A$ is less frequent than varus $O A$ and causes of valgus $O A$ include medial ligament instability, flat foot and posttraumatic situations, e.g. fractures of the fibula or lateral tibial plafond. The importance of the mechanical axis is generally accepted in orthopedic surgery. In cases of implantation of total ankle replacements the normal biomechanics need to be restored in order to have a correct and pain-free functioning total ankle replacement both in the short and long-term. The two most important criteria are (1) an anterior tibio-talar angle of about $90^{\circ}$ and (2) a neutral hindfoot position. The hindfoot position is measured with the hindfoot alignment view according to Saltzman. In this view, healthy feet are in neutral or minimal varus position of $1-2^{\circ}$ and not in a valgus position as generally assumed. The following operative steps are performed depending on the degree and localization of the valgus deformity: (1) total ankle replacement, (2) supramalleolar or (3) inframalleolar osteotomy/arthrodesis, (4) medial ligament repair, (5) fibula osteotomy and (6) syndesmotic reconstruction.

\section{Keywords}

Total ankle arthroplasty · Valgus · Ankle osteoarthritis · Syndesmoplasty · Arthrodesis 
werden kann, während hingegen bei einer größeren Deformität in der Frontalebene ein großes Risiko für ein rasches mechanisches Versagen der Prothese besteht [15, 26,27 ]. Doets et al. [10] präsentierten eine 8-Jahres-Überlebensrate von 90\% für Fälle mit präoperativ normalem Alignement gegenüber von $84 \%$ bei einer Deformität in der Frontalebene $>10^{\circ}$. Dies hat dazu geführt, dass manche Autoren eine Fehlstellung $>10^{\circ}$ generell als Kontraindikation für eine OSG-Prothesen-Implantation sehen.

Bei einer Valgus-OSG-Arthrose empfiehlt sich vor dem Einbau der OSG-Prothese eine suffiziente präoperative Röntgenuntersuchung mit Röntgen im Stehen des Sprunggelenks (OSG a.-p., Fuß a.-p. und lateral, Saltzman-Aufnahme) sowie der gesamten unteren Extremität (Orthoradiogramm), da die OSG-Arthrose häufig mit einem Genu valgum vergesellschaftet sein kann (- Abb. 2). In einem solchen Fall sollte die Deformität sowohl im OSG wie auch auf Niveau des Knies angegangen werden ( $\bullet$ Abb. 3).

\section{- Die Normalisierung der Biomechanik bei einer Valgus-OSG-Arthrose ist individuell festzulegen.}

Zur Bestimmung der Deformität ist zwingend eine Saltzman-Aufnahme („hindfoot alignement view") nötig, da die visuelle Einschätzung am Patienten nur zu $50 \%$ mit den gemessenen Werten übereinstimmt und eine große Varianz aufweist [12]. Je nach Ausmaß der Valgusfehlstellung und deren Ätiologie können folgende Zusatzeingriffe notwendig werden (• Abb. 6):

- varisierende supramalleoläre Korrekturosteotomie - häufig mediale zuklappende Osteotomie,

- verlängernde und häufig auch derotierende Osteotomie der Fibula,

- varisierende Osteotomie des Kalkaneus mittels medialisierender Verschiebe- oder lateraler Verlängerungsosteotomie,

- Rekonstruktion des medialen OSGBandapparats [25],

- varisierende Subtalar- oder Triplearthrodese.
Bei einer schweren Valgusfehlstellung aufgrund eines schweren Valgustibiaplafonds sollte chronologisch bei der Operation zuerst am Ort der größten Deformität korrigiert, d. h. zuerst die varisierende supramalleoläre Korrekturosteotomie durchgeführt und erst danach die OSG-Prothese eingebaut werden. Je nach Notwendigkeit sollten sich danach die Kalkaneusosteotomie oder die USG-/Triplearthrodese, Fibulaosteotomie und/oder mediale Bandplastik anschließen. Bei einer schweren Valgusfehlstellung aufgrund eines schweren Valgusrückfußes (unteres Sprunggelenk [USG], Chopart) sollten zuerst die varisierende Subtalar-/Triplearthrodese realisiert und danach die OSG-Prothese eingebaut werden (• Abb. 4), gefolgt von der häufig notwendigen medialen OSG-Bandplastik [25] und in manchen Fällen einer Fibulaverlängerungsosteotomie. Bei einer moderaten Valgusfehlstellung wird zuerst die OSG-Prothese eingebaut und danach die varisierende Kalkaneusosteotomie vorgenommen (Rückfuß ohne Arthrose) oder USG-/Triplearthrodese (bei kombinierter Rückfußarthrose), oder eine Fibulaverlängerungsosteotomie (- Abb. 5) sowie die mediale Bandplastik [25] ergänzt (• Abb. 6).

\section{Problem OSG-Prothesen bei persistierender Valgusfehlstellung}

Die Wichtigkeit biomechanischer Achsen in der Orthopädie ist weitgehend akzeptiert. So ist für das Knie beschrieben, dass schon kleine Achsabweichungen bei der prothetischen Versorgung gravierende Folgen für das Implantatüberleben und das klinische Ergebnis haben können [18]. Frigg et al. [13] wiesen nach, dass die Saltzman-Aufnahme am besten das dynamische Belastungsmuster vorhersagen konnte. Auch ergaben sich die physiologischsten Werte für ein Alignement, das genau neutral oder sogar leicht varisch in dieser Aufnahme war.

- Weitere Studien sind nötig, um Achsabweichungen und ihre Auswirkungen auf das prothetisch versorgte OSG weiter zu untersuchen.

Heute betonen die meisten Autoren, dass Achsfehlstellungen in der Frontalebene
Hier steht eine Anzeige.

Springer 
Hier steht eine Anzeige.

算 Springer 

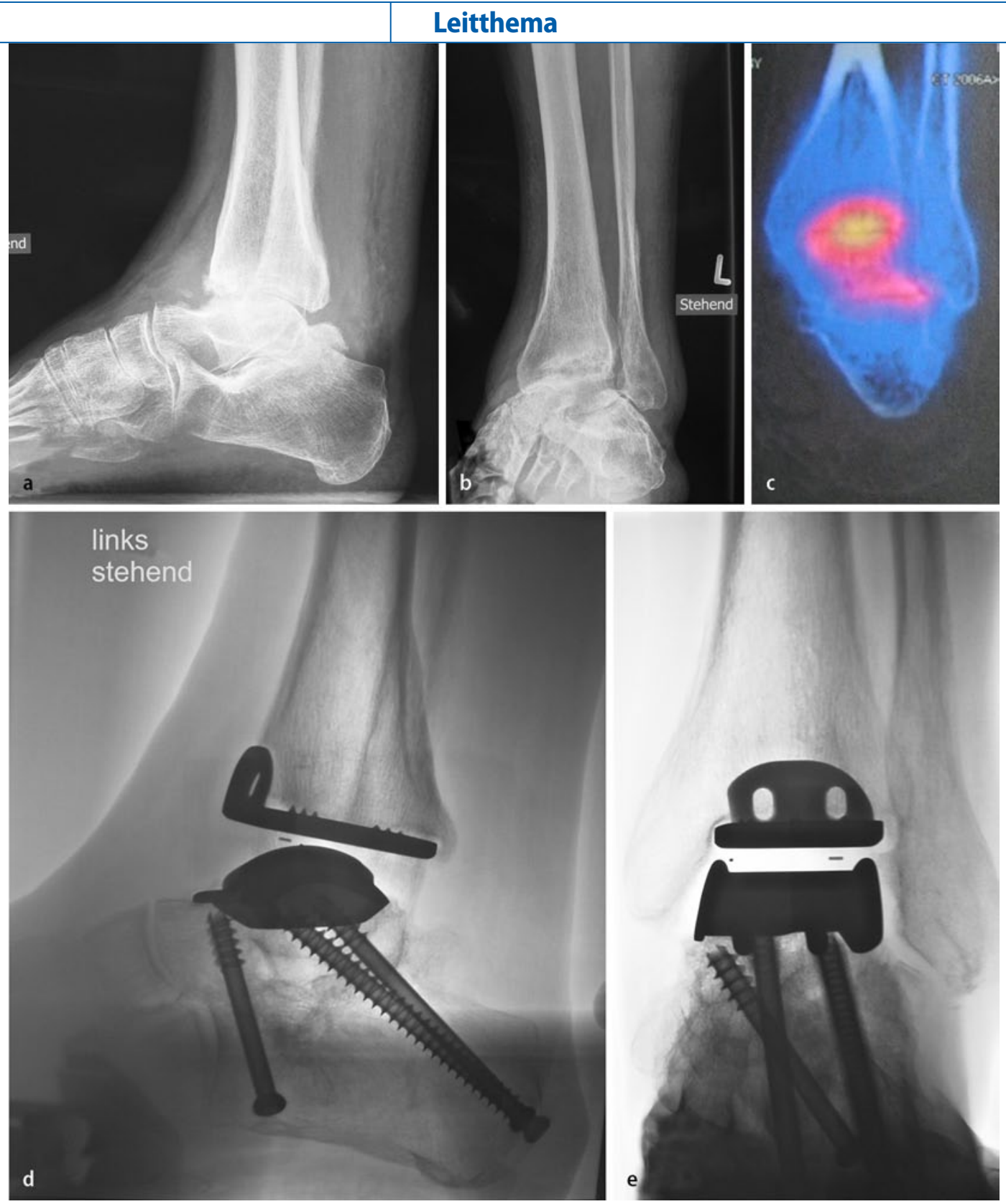

Abb. $4<$ Valgus-OSG-Arthrose mit schwerem Valgusrückfuß Der 65-jährige Patient litt an einer progredienten Gelenkdegeneration mit zunehmendem Valguskollaps im OSG wie auch USG bei bekannter villonodulärer Synovialitis. Nach Normalisierung des Rückfußalignements mittels einer varisierenden Subtalararthrodese erfolgte die Implantation einer OSG-Prothese in korrekter Position. OSG oberes, USG unteres Sprunggelenk

korrigiert werden sollten, um ein gutes Langzeitergebnis zu erhalten. Wird dies nicht berücksichtigt, drohen eine einseitig vermehrte Randbelastung der Prothesenkomponenten, ein Polyethylenverschleiß, eine Subluxation des Polyethylengleitkernes und ein frühzeitiges Prothesenversagen $[6,7,11,14,27]$.

\section{Fazit für die Praxis}

Damit eine OSG-Prothese kurz- wie auch langfristig korrekt und schmerzfrei funktionieren kann, muss die Biomechanik entsprechend den Prinzipien der mechanischen Achse wiederhergestellt werden. Die wichtigsten Richtlinien sind dabei
(1) ein anteriorer tibiotalarer Winkel von etwa $90^{\circ}$ und (2) eine neutrale Rückfußstellung in der Rückfußaufnahme nach Saltzman. Eine moderate Valgusarthrose bis $10^{\circ}$ kann direkt mit der korrekten Schnittführung bei der Implantation der OSG-Prothese korrigiert werden. Sollte dies intraoperativ nicht ausreichen, erfolgt zusätzlich eine Korrektur des Rückfußes, der Bänder und eventuell der Fibula (- Abb. 6). Bei einer schweren Valgusarthrose über $10^{\circ}$ muss zuerst das Alignement korrigiert werden - je nach Lokalisation der Deformität zuerst an der Tibia oder am Rückfuß. Anschließend erfolgt in derselben Operation der Einbau der OSG-Prothese und danach eventu- ell eine Vervollständigung der Valguskorrektur mit einer medialen Bandplastik und/oder Fibulaosteotomie (• Abb. 6).

\section{Korrespondenzadresse}

Prof. Dr. Dr. V. Valderrabano

Abteilung Orthopädie,

Universitätsklinikum Basel

Spitalstr. 21, CH-4031 Basel

Schweiz

vvalderrabano@uhbs.ch

Interessenkonflikt. Der korrespondierende Autor gibt an, dass kein Interessenkonflikt besteht. 

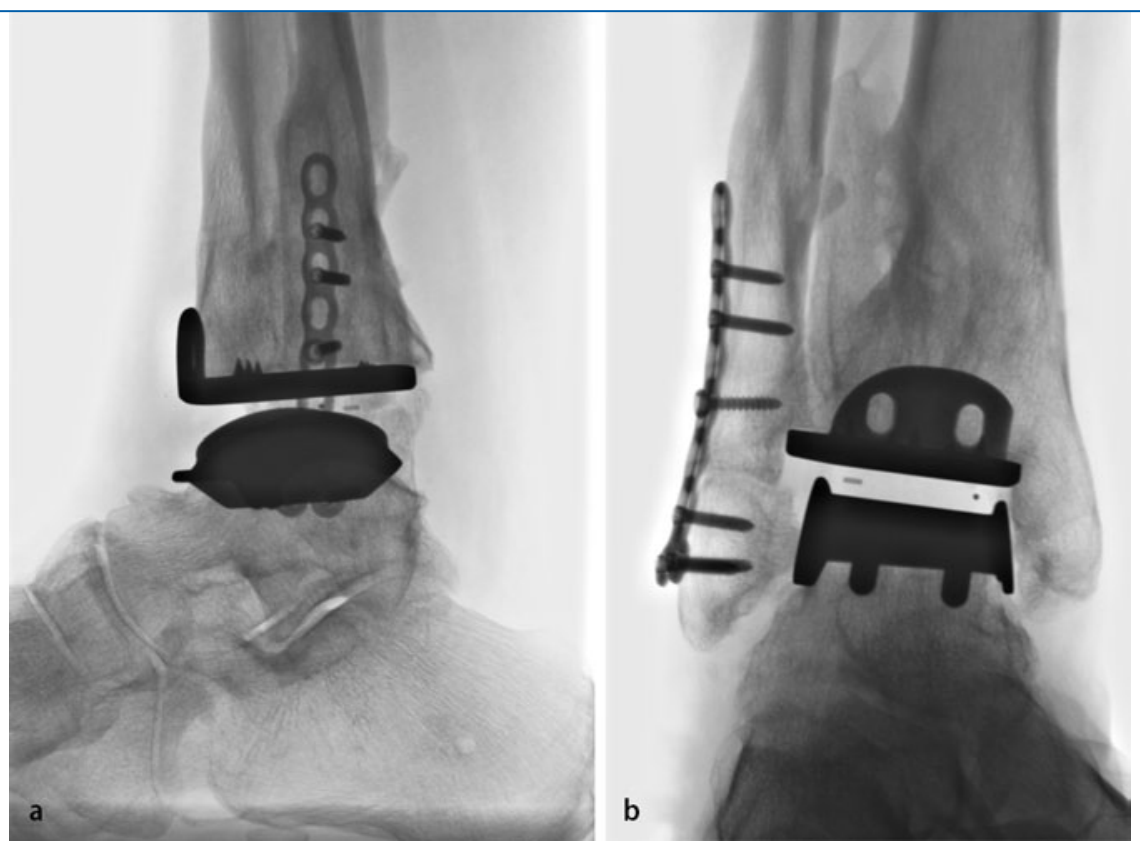

Abb. $5 \Delta$ Korrektur der Deformität über eine derotierende, verlängernde Fibulaosteotomie beim Fall aus Abb. 3. a Seitliche, b a.-p.-Projektion

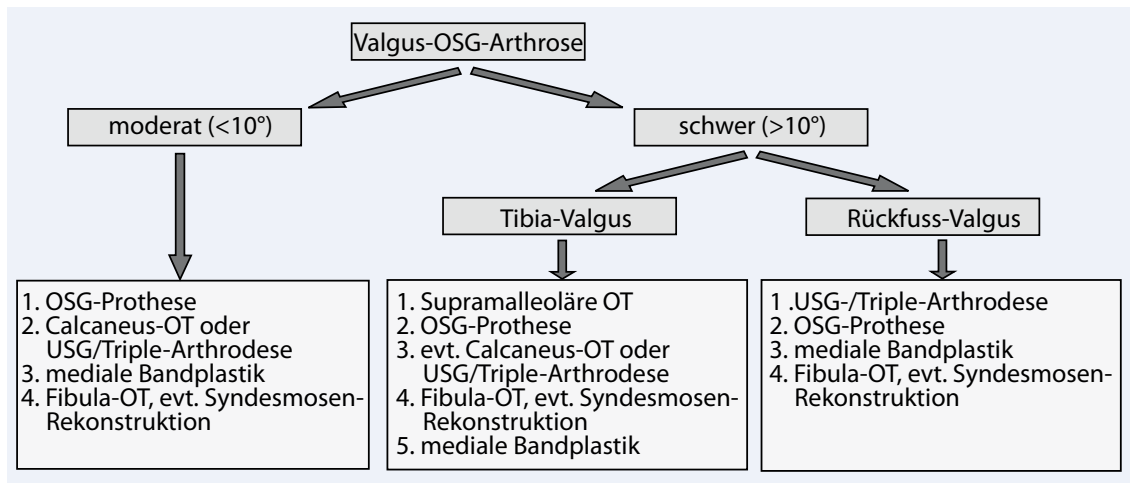

Abb. $6 \Delta$ Behandlungsalgorithmus der Deformitätenkorrektur in Abhängigkeit von der Schwere und Lokalisation der Deformität. OT Osteotomie

\section{Literatur}

1. Backer M, Kofoed H (1989) Passive ankle mobility. J Bone Joint Surg 71-B:696-698

2. Bohay D, Anderson J (2003) Stage IV posterior tibial tendon insufficiency: the tilted ankle. Foot Ankle Clin 8:619-636

3. Brinker M (2001) Review of orthopaedic trauma. Saunders-Elsevier, Philadelphia

4. Buck P, Morrey BF, Chao EY (1987) The optimum position of arthrodesis of the ankle. A gait study of the knee and ankle. J Bone Joint Surg [Am] 69:1052-1062

5. Clarke H, Michelson J, Cox Q, Jinnah R (1991) Tibio-talar stability in bimalleolar ankle fractures: a dynamic in vitro contact area study. Foot Ankle Int 11:222-227

6. Coetzee J (2008) Management of varus or valgus ankle deformity with ankle replacement. Foot Ankle Clin North Am 13:509-520

7. Conti S, Wong Y (2001) Complications of total ankle replacement. Clin Orthop Relat Res 391:105114
8. Coughlin M, Mann R, Saltzman C (2007) Surgery of the foot and ankle. Mosby-Elsevier, Philadelphia

9. De Deland J, Asla R, Segal A (2004) Reconstruction of the chronically failed deltoid ligament: a new technique. Foot Ankle Int 25:795-799

10. Doets H, Brand R, Nelissen R (2006) Total ankle arthroplasty in inflammatory joint disease with use of two mobile-bearings designs. J Bone Joint Surg 88-A:1272-1284

11. Doets H, Plaat L, Van der Klein J (2008) Medial malleolar osteotomy for the correction of varus deformity during total ankle arthroplasty: results in 15 ankles. Foot Ankle Int 29:171-177

12. Frigg A, Nigg B, Davis E et al (2010) Does alignment in the hindfoot radiograph influence dynamic foot-floor pressures in ankle and tibiotalocalcaneal fusion? Clin Orthop Relat Res 468:33623370

13. Frigg A, Nigg B, Hinz L et al (2010) Clinical relevance of hindfoot alignment view in total ankle replacement. Foot Ankle Int 31:871-879

14. Haskell A, Mann R (2004) Ankle arthroplasty with preoperative coronal plane deformity: short term results. Clin Orthop 424:98-103
15. Hennessy M, Molloy A, Wood E (2008) Management of the varus arthritic ankle. Foot Ankle Clin North Am 12:417-442

16. Horisberger M, Valderrabano V, Hintermann B (2009) Posttraumatic ankle osteoarthritis after ankle-related fractures. J Orthop Trauma 23:60-67

17. Johnson JE, Lamdan R, Granberry WF et al (1999) Hindfoot coronal alignment: a modified radiographic method. Foot Ankle Int 20(12):818-825

18. Miller M (2004) Review of orthopaedics. SaundersElsevier, Philadelphia

19. Pagenstert G, Hintermann B, Barg A et al (2007) Realignment surgery as alternative treatment of varus and valgus ankle osteoarthritis. Clin Orthop Relat Res 462:156-168

20. Saltzman CL, El-Khoury GY (1995) The hindfoot alignment view. Foot Ankle Int 16(9):572-576

21. Tarr R, Resnick C, Wagner K, Sarmiento A (1985) Changes in tibiotalar joint contact areas following experimentally induced tibial angular deformities. Clin Orthop Relat Res 199:72-80

22. Ting A, Tarr R, Sarmiento A et al (1987) The role of subtalar motion and ankle contact pressure changes from angular deformities of the tibia. Foot Ankle 7:290-299

23. Valderrabano V, Hinterman B, Horisberger M, Fung $T$ (2006) Ligamentous posttraumatic ankle osteoarthritis. Am J Sports Med 34:612-620

24. Valderrabano V, Horisberger $M$, Russell I et al (2009) Etiology of ankle osteoarthritis. Clin Orthop Relat Res 467:1800-1806

25. Valderrabano V, Wiewiorski M, Frigg A et al (2007) Chronic ankle instability. Unfallchirurg 110:691699

26. Wood P, Deakin S (2003) Total ankle replacement: the results in 200 ankles. J Bone Joint Surg [Br] 85B:334-341

27. Wood P, Sutton C, Mishra V, Suneja R (2009) A randomized controlled trial of two mobilebearing total ankle replacements. J Bone Joint Surg 91-B:69-74 